\title{
Aula Mix e treinamento resistido: comparação da influência de treinamento sobre valências físicas*
}

\section{Class mix and resistance training: comparison of training influence on physical valences}

Arthur Alves da Silva' Marcela Cardoso Costa de Oliveira ${ }^{2}$ Giuliano Roberto da Silva ${ }^{3}$
* Recebido em: 02/06/14 Aprovado em: 13/10/14

1 Universidade José do Rosário Vellano - UNIFENAS - Alfenas - MG - Brasil. Email - arthur_predador@hotmail.com.

2 Universidade José do Rosário Vellano - UNIFENAS - Alfenas - MG - Brasil. Email -marcela_fitness@hotmail.com.

3 Universidade José do Rosário Vellano - UNIFENAS - Alfenas - MG - Brasil; Universidade Vale do Rio Verde - UNINCOR - Três Corações - MG - Brasil; Faculdade Presbiteriana Gammon - FAGAMMON - Lavras - MG Brasil.Email - giumusc@gmail.com.

\section{Resumo}

O estudo objetivou comparar aula mix e treinamento resistido, avaliando quais teriam maior influência no percentual de gordura, flexibilidade e força. Foram elencadas 20 universitárias entre 20 e 30 anos, em dois grupos, $\mathrm{n} 1=10$ praticantes de aula mix (jump, cycle indoor, step e ginástica localizada) e $\mathrm{n} 2=10$ praticantes de treinamento resistido. Os treinos foram realizados durante 8 semanas em 3 sessões de 60 minutos, em dias alternados. Os métodos de avaliação foram: "Sargent Jump", arremesso de Medicine Ball, teste no banco de Wells e mensuração das sete dobras cutâneas. A aula mix proporcionou melhor resultado no percentual de gordura e flexibilidade, e o treinamento resistido, apresentou melhor resultado no ganho de força de membro superior e inferior, considerando $\mathrm{p} \leq 0,05$. Porém, independente da modalidade escolhida, às variáveis estudadas apresentou mudanças positivas, logo a prática de exercícios físicos garante promoção de saúde e qualidade de vida.

Palavras-chave: Treinamento resistido. Aula Mix. Percentual de gordura. Flexibilidade. Força.

\begin{abstract}
The study aimed to compare Class Mix and Resistance Training evaluating what would have greater influence on the percentage of fat, flexibility and strength. Were listed 20 universities from 20 to 30 years, in two groups, $\mathrm{n} 1=10$ practitioners Class Mix (jump, indoor cycle, step and located gymnastics) and $\mathrm{n} 2=10$ practitioners of Resistance Training. Training sessions were conducted for 8 weeks for three 60 minute sessions on alternate days. The assessment methods were "Sargent Jump", throwing Medicine Ball, test the bank Wells and measurement of seven skinfolds. The Class Mix provided better results in the percentage of fat and flexibility, and the Resistance Training, showed better results in the upper limb strength gain and lower, considering $\mathrm{p} \leq 0.05$. However, regardless of the chosen mode, the variables studied showed positive changes, so the physical exercise ensures health promotion and quality of life.
\end{abstract}

Keywords: Resistance Training. Classmix. Fat percentage. Strength. 


\section{Introdução}

Atualmente, já não há dúvidas de que a prática de atividade física contribui diretamente para a manutenção e promoção da saúde, ao melhorar a resistência, força muscular, equilíbrio e agilidade e, consequentemente, reduzir os riscos de problemas de saúde, além de contribuir, também, para a saúde mental.

A atividade física é tudo aquilo que implica movimento, força ou manutenção da postura corporal contra a gravidade e se traduza em consumo de energia. Já o exercício físico é uma atividade repetitiva e estereotipada, que visa à obtenção de um objetivo concreto. As diferenças entre atividade física e exercício físico, a partir da intencionalidade do movimento, refere-se ao fato de o exercício físico consistir em subgrupo das atividades físicas, planejado, estruturado e repetitivo, tendo como propósito a manutenção ou a otimização do condicionamento físico, considerando-se também que este constitui um instrumento de promoção à saúde, e deve ser cuidadosamente prescrito, baseando-se em evidências científicas (BARATA, 1997; CARVALHO et al., 2004)

O exercício regular não significa apenas atividade desportiva, de lazer ou competitiva, mas também outras atividades diárias, como por exemplo, relacionadas com certas áreas de trabalho manual, portanto, a prática de exercício é aconselhada a fim de preservar o bem- estar físico, psíquico e social da população, ou seja, é fundamental no que se refere à saúde, pois objetiva o desenvolvimento de aptidões físicas e habilidades motoras por meio de atividades aeróbias (exercício produzido com baixa intensidade num longo espaço de tempo, tais como a Aula Mix) ou anaeróbias (exercício produzido num espaço de tempo breve e com muita intensidade, tais como o treinamento resistido) desenvolvidas por sujeitos que se diferem na sua condição e capacidades físicas, é considerado fundamental no que se refere à saúde (HORTA; BARATA, 1997; GONÇALVES; VILARTA, 2004).

A atividade física regular é considerada de grande importância para a saúde, sendo reconhecida por seus efeitos saudáveis nos praticantes, sendo possível relacioná-la a alterações positivas para combater ou prevenir o aparecimento de diversas doenças, tais como: doenças cardiovasculares, obesidade, diabetes, osteoporose, entre outras, e também promover bem-estar psicológico, me- lhorar a autoestima, causar redução no risco de desenvolver doenças cardíacas, e melhorar a disposição para tarefas diárias (WEINECK, 2005; SANTOS; FISCHER, 2007).

Dentre os tipos de exercícios físicos oferecidos, Fleck e Kraemer (2006), apontam que muitas atividades têm o propósito de desenvolver melhor aptidão física, além de oferecer alterações positivas no percentual de gordura corporal, dentre essas atividades, está o treinamento resistido e os exercícios aeróbicos.

O treinamento resistido é um tipo de exercício resistido com pesos (pesos livres ou equipamentos), no qual ocorrem contrações da musculatura esquelética de um seguimento corporal contra uma resistência externa, sendo considerados os mais completos entre as formas de treinamentos físicos, além de trazer benefícios como, alterações na composição corporal, além de força e hipertrofia muscular (SANTARÉM, 1999; MATSUDO, 1997; FLECK; KRAEMER, 2006; UCHIDA et al., 2006).

Os exercícios conhecidos como aeróbicos geralmente realizados em Aula Mix, são movimentos voluntários dependentes de oxigênio, em que o sistema cardiorrespiratório é o que determina a resistência e a adaptação física ao exercício, em que pode ser aplicado a todas as faixas etárias e a diferentes níveis de condicionamentos (HOLLMANN; HETTINGER, 1983; VILLALBA et. al., 2005). A aula mix pode ser composta por jump, cycle indoor, step e ginástica localizada (ANJOS et al., 2006).

Portanto, a proposta deste estudo trouxe uma situação nova à medida que esteve relacionada com a meta de Promoção de Saúde, visto que, mesmo que haja na literatura estudos comprovando que há mudanças no percentual de gordura, flexibilidade e força por meio da prática de treinos aeróbicos e anaeróbicos, esta nova proposta trouxe um diferencial específico, uma vez que ainda não existia na literatura a comparação dessas duas modalidades que foram escolhidas (aula mix e treinamento resistido), para averiguar possíveis mudanças nas valências físicas escolhidas, citadas acima.

Logo, o objetivo deste estudo consiste em comparar as valências físicas por meio de protocolo de treino das modalidades: treinamento resistido e aula mix (jump, cycle indoor, step e ginástica localizada) em mulheres jovens, a fim de comprovar qual destas tem maior eficiência na perda de percentual de gordura, no ganho de força e de flexibilidade. 


\section{Metodologia}

Trata-se de um estudo longitudinal uma vez que os pesquisadores coletaram os dados em um curto espaço de tempo. E também um estudo descritivo, com abordagem quantitativa, pois houve análise comparativa dos resultados nas variáveis de aptidão física: percentual de gordura, força e flexibilidade, por meio das modalidades aula mix e treinamento resistido a fim de comparar como tais variáveis se comportam em ambas as modalidades e, com isso, averiguar qual destas atividades físicas tem maior eficiência fisiológica e morfológica sobre as variáveis propostas.

Desse modo, o estudo foi constituído com uma amostra de 20 mulheres entre 20 e 30 anos de idade, sendo um grupo com $n=10$ praticantes de aula mix e outro grupo com $\mathrm{n}=10$ praticantes de treinamento resistido.

Como critério de inclusão, levou-se em consideração mulheres com idade entre 20 e 30 anos, universitárias, não praticantes de atividade física e que se dispuseram a participar do estudo, e, como critério de exclusão, mulheres que não se encaixaram nas preposições anteriores. O recrutamento das participantes ocorreu por meio de convite verbal no campus da universidade.

Todas as participantes do estudo foram devidamente informadas sobre os testes, os métodos de treinamento e possíveis benefícios ou riscos. Estando de acordo, as participantes assinaram o Termo de Consentimento Livre e Esclarecido, aprovado pelo Comitê de Ética em Pesquisa da Universidade José do Rosário Vellano (UNIFENAS), conforme Resolução no 466 de dezembro de 2012 do Conselho Nacional de Saúde (CNS), que trata das diretrizes e normas regulamentadoras de pesquisas envolvendo seres humanos.

As participantes do estudo foram, então, submetidas às avaliações antes de iniciar o programa de treinamento, que perdurou por 8 semanas. A frequência do treinamento foi controlada pelos pesquisadores por meio de uma ficha de presença, no qual continha o nome, o horário do treino, em que cada participante teve que assinar essa ficha em cada sessão de treinamento, durante todo o protocolo realizado.

O protocolo de treino ocorreu da seguinte forma: o treinamento resistido foi realizado em três sessões de 60 minutos de duração durante a semana, em dias alternados, em que as sessões também se alternavam entre os treinos denominados A, B, ou C. Enquanto a aula mix (ginástica localizada, jump, step e cycle indoor) foi realizada em três sessões durante a semana, em dias alternados, com 60 minutos de duração, a qual a cada sessão era realizada um tipo de modalidade da aula mix (jump, step ou cycle indoor) com duração de 25 minutos, e mais 35 minutos de ginástica localizada, visando à resistência e à força, alternando-se também entre os treinos denominados A, B, ou C.

Para aferir medidas de dobras cutâneas, foi usado o protocolo de Pollock, Rocha e Wilmore (1993) (Tabela 1), o qual usa sete dobras cutâneas, entre elas: subescapular, tricipital, peitoral, axilar média, suprailíacas, abdominal e coxa. Para pinçar as dobras, foi usado um adipômetro clínico Sanny ${ }^{\bullet}$, além de fita métrica para medir circunferências corporais e altura, e balanças para averiguar o peso (POLLOCK; ROCHA; WILMORE, 1993).

Tabela 1 - Classificação de percentual de gordura para mulheres

\begin{tabular}{|c|c|c|c|c|c|}
\hline \multicolumn{6}{|c|}{ Classificação do percentual de gordura (\%G) para mulheres } \\
\hline Nível/Idade & 18 a 25 & 26 a 35 & 36 a 45 & $46-55$ & $56-65$ \\
\hline Excelente & 13 a $16 \%$ & 14 a $16 \%$ & 16 a $19 \%$ & 17 а $21 \%$ & 18 a $22 \%$ \\
\hline Bom & 17 а $19 \%$ & 18 a $20 \%$ & 20 a $23 \%$ & 23 a $25 \%$ & 24 a $26 \%$ \\
\hline Acima da Média & 20 а $22 \%$ & 21 а $23 \%$ & 24 a $26 \%$ & 26 a $28 \%$ & 27 a $29 \%$ \\
\hline Média & 23 а $25 \%$ & 24 а $25 \%$ & 27 а $29 \%$ & 29 а $31 \%$ & 30 a $32 \%$ \\
\hline Abaixo da Média & 26 a $28 \%$ & 27 а $29 \%$ & 30 а $32 \%$ & 32 а $34 \%$ & 33 а $35 \%$ \\
\hline Ruim & 29 a $31 \%$ & 31 а $33 \%$ & 33 a $36 \%$ & 35 a $38 \%$ & 36 a $38 \%$ \\
\hline Muito Ruim & 33 а $43 \%$ & 36 а $49 \%$ & 38 a $48 \%$ & 39 а $50 \%$ & 39 a 49\% \\
\hline
\end{tabular}

Fonte: Pollock, Rocha e Wilmore (1993)

Para avaliar flexibilidade, foi realizado o teste de sentar e alcançar no banco. (Tabela 2). Para realizá-lo é necessário o uso do banco específico, em que o avaliado deve estar sentado, com os pés devidamente apoiados ao banco e os joelhos estendidos. Na sequência, o tronco deve ser flexionado a frente, buscando sempre a maior quantidade de centímetros possíveis. Os joelhos do avaliado devem ser apoiados pelo avaliador para evitar sua flexão (WELLS; DILLON, 1952; CMSFAS,1987).

Tabela 2 - Teste de flexibilidade sentar e alcançar

\begin{tabular}{lcccccc}
\hline \multicolumn{6}{l}{ Classificação do teste de sentar e alcançar - Feminino - com banco (em centímetros) } \\
\hline Idade & $15-19$ & $20-29$ & $\mathbf{3 0 - 3 9}$ & $\mathbf{4 0 - 4 9}$ & $\mathbf{5 0 - 5 9}$ & $\mathbf{6 0 - 6 9}$ \\
Excelente & $>43$ & $>41$ & $>41$ & $>38$ & $>39$ & $>35$ \\
Acima da Média & $38-42$ & $37-40$ & $36-40$ & $34-37$ & $33-38$ & $31-34$ \\
Média & $34-37$ & $33-36$ & $32-35$ & $30-33$ & $30-32$ & $27-30$ \\
Abaixo da Média & $29-33$ & $28-32$ & $27-31$ & $25-29$ & $25-29$ & $23-26$ \\
Ruim & $<28$ & $<27$ & $<26$ & $<24$ & $<24$ & $<22$ \\
\hline
\end{tabular}

Fonte: Wells e Dillon (1952) e CMSFAS (1987)

A avaliação de força de membro superior foi feita por meio do teste de arremesso de Medicine Ball (Tabela 
3). Esse teste é executado da seguinte forma: uma trena é fixada ao solo perpendicular a uma parede. $\mathrm{O}$ aluno senta-se com os joelhos estendidos, e pernas unidas e as costas completamente apoiadas à parede. Segura a medicine Ball junto ao peito, e, ao sinal do avaliador, o aluno deverá lançar a bola na maior distância possível, mantendo as costas apoiadas na parede. A distância do arremesso será registrada a partir do ponto zero até o local em que a bola tocou o solo pela primeira vez (MARINS; GIANNICHI, 1998).

Tabela 3 - Arremesso de Medicine Ball

\begin{tabular}{cc}
\hline \multicolumn{2}{c}{ Classificação de Arremesso de Medicine Ball } \\
\hline Avançado & Acima $428 \mathrm{~cm}$ \\
Intermediário avançado & $367-427 \mathrm{~cm}$ \\
Intermediário & $214-316 \mathrm{~cm}$ \\
Iniciante avançado & $123-213 \mathrm{~cm}$ \\
Iniciante & $0-122 \mathrm{~cm}$ \\
\hline
\end{tabular}

Fonte: Marins e Giannichi (1998)

Foi utilizado para o teste de força de membro inferior o teste de impulsão vertical "Sargent Jump" (Tabela 4). A realização desse teste ocorreu da seguinte forma: uma tábua foi fixada na parede a um metro de altura do solo, contendo um metro e cinquenta centímetros de comprimento, com trinta centímetros de largura, demarcada em centímetros. $\mathrm{O}$ executante com os pés juntos em uma linha demarcada no solo, passar pó de giz nas pontas dos dedos da mão dominante. Deve saltar a máxima altura, fazendo uma marca na tábua com os dedos "sujos” de giz, procurando alcançar o ponto mais alto possível. Não é permitido andar ou tomar distância para saltar (MARINS; GIANNICHI, 1998).

Tabela 4 - Impulsão vertical

\begin{tabular}{cc}
\hline \multicolumn{2}{c}{ Classificação da impulsão vertical } \\
\hline Excelente & $>33$ \\
Acima da Média & $26-33 \mathrm{~cm}$ \\
Média & $16-25 \mathrm{~cm}$ \\
Abaixo da Média & $06-15 \mathrm{~cm}$ \\
Fraco & $2,5-5 \mathrm{~cm}$ \\
\hline
\end{tabular}

Fonte: Marins e Giannichi (1998)

A responsabilidade pela elaboração, aplicação e monitoramento dos testes foi dos pesquisadores, supervisionados pelo orientador da pesquisa.

Para o estudo relatado neste artigo, os resultados
Student, considerando $\mathrm{p} \leq 0,05$.

\subsection{Programas de treinamento}

O programa de treinamento na aula mix foi realizado da seguinte forma: 3 sessões na semana, em que cada dia era utilizado um treino aeróbico, alternando entre jump, cycle indoor e step, todos com duração de 25 minutos e, em seguida, eram realizados exercícios localizados, visando à resistência, com duração de 35 minutos ou força, também com duração de 35 minutos, em que em cada sessão de treino alternava-se entre as sequências A, B ou C (Quadro 1).

Para o treinamento resistido, foi utilizado o método alternado por segmento Bittencourt (1986), objetivando ganho de resistência e força explosiva (Quadro 2), em que, em cada sessão de treino (3 por semana), alternava-se entre as sequências $\mathrm{A}, \mathrm{B}$ ou C. Portanto, os treinos foram de força explosiva com 04 séries de 06 repetições, com intervalo de 1 a 2 minutos, e o de resistência com 03 séries de 15 repetições com o intervalo de 45 segundos a 1 minuto.

Quadro 1 - Programa de treinos para aula mix

\begin{tabular}{|c|c|c|c|c|}
\hline \multicolumn{5}{|c|}{ AULA MIX } \\
\hline Modalidade & $\begin{array}{c}\text { № de sessões } \\
\text { (semana) }\end{array}$ & Aquecimento & Parte principal & Intensidade \\
\hline Jump & 1 & $\begin{array}{l}5 \text { minutos } \\
\text { no solo }\end{array}$ & $\begin{array}{c}25 \text { minutos de chutes, } \\
\text { polichinelo, elevação } \\
\text { de joelhos, corridas } \\
\text { e movimentos de } \\
\text { braços }\end{array}$ & 140 bpm $^{*}$ \\
\hline Cycle Indoor & 1 & $\begin{array}{l}5 \text { minutos } \\
\text { pedalando } \\
\text { sentada }\end{array}$ & $\begin{array}{c}10 \text { minutos } \\
\text { pedalando } \\
\text { intervalado, em pé e } \\
\text { sentado; } 2 \text { minutos } \\
\text { pedalando sentado; } 6 \\
\text { minutos intervalado e } \\
2 \text { minutos pedalando } \\
\text { sem carga }\end{array}$ & 140 bpm* \\
\hline Step & 1 & $\begin{array}{l}5 \text { minutos } \\
\text { marchando no } \\
\text { solo e subindo } \\
\text { e descendo da } \\
\text { plataforma }\end{array}$ & $\begin{array}{c}20 \text { a } 25 \text { minutos, } \\
\text { subidas e descida } \\
\text { frontais laterais e ao } \\
\text { redor da plataforma. }\end{array}$ & 132 bpm* \\
\hline \multicolumn{5}{|c|}{ Treino localizado A (AULA MIX) - RESISTÊNCIA } \\
\hline $\begin{array}{c}N^{N} \text { de sessões } \\
\text { (Semana) }\end{array}$ & Exercícios & $\mathrm{N}^{0}$ de séries & $\mathrm{N}^{0}$ de repetições & $\begin{array}{l}\text { Variação } \\
\text { de carga }\end{array}$ \\
\hline 1 & $\begin{array}{c}\text { Supino reto com } \\
\text { halter, remada } \\
\text { unilateral, } \\
\text { elevação frontal, } \\
\text { panturrilha, } \\
\text { glúteo } 4 \text { apoios. }\end{array}$ & 3 & 15 & 4 a $8 \mathrm{Kg}^{*}$ \\
\hline \multicolumn{5}{|c|}{ Treino localizado B (AULA MIX) - RESISTÊNCIA } \\
\hline 1 & \begin{tabular}{|} 
Rosca direta, \\
tríceps testa, \\
adução, flexão de \\
tronco.
\end{tabular} & 3 & 15 & $\begin{array}{c}4 \text { a } 6 \mathrm{Kg}^{*} \\
\text { Flexão de } \\
\text { Tronco: Livre }\end{array}$ \\
\hline
\end{tabular}




\begin{tabular}{|c|c|c|c|c|}
\hline \multicolumn{5}{|c|}{ Treino localizado C (AULA MIX) - RESISTÊNCIA } \\
\hline 1 & $\begin{array}{c}\text { Extensão de pernas, } \\
\text { flexão de pernas, } \\
\text { abduçãa,avanç̧o, flexão } \\
\text { de coxa. }\end{array}$ & 3 & 15 & $\begin{array}{l}\text { 6 a 8 8 } \mathrm{Kg}^{*} \\
\text { Flexão de } \\
\text { coxa: Livre }\end{array}$ \\
\hline \multicolumn{5}{|c|}{ Treino localizado A (AULA MIX) - FORÇA } \\
\hline 1 & $\begin{array}{c}\text { Agachamento, } \\
\text { extensão de pernas, } \\
\text { flexão de pernas, } \\
\text { adução, flexão de coxa. }\end{array}$ & 4 & 6 & 6а $10 \mathrm{Kg}^{*}$ \\
\hline \multicolumn{5}{|c|}{ Treino localizado B (AULA MIX) - FORÇA } \\
\hline 1 & $\begin{array}{l}\text { Crucifixo, remada } \\
\text { inclinada, elevaçãa } \\
\text { lateral, glúteo } 4 \text { apoios. }\end{array}$ & 4 & 6 & 6 a $8 \mathrm{Kg}^{*}$ \\
\hline \multicolumn{5}{|c|}{ Treino localizado C (AULA MIX) - FORÇA } \\
\hline \multirow[t]{2}{*}{1} & $\begin{array}{l}\text { Rosca alternada, tríceps } \\
\text { francês, abdução, } \\
\text { panturrilha. }\end{array}$ & 4 & 6 & \multirow[t]{2}{*}{4 a $20 \mathrm{Kg}^{*}$} \\
\hline & Abdominal crunch & 4 & 20 & \\
\hline
\end{tabular}

Legenda: ${ }^{\star}$ bpm: batidas por minuto; ${ }^{\star} \mathrm{kg}$ : kilograma. Fonte: do autor

Quadro 2 - Programa de treinos para treinamento resistido

\begin{tabular}{|c|c|c|c|c|}
\hline \multicolumn{5}{|c|}{ TREINAMENTO RESISTIDO } \\
\hline \multicolumn{5}{|c|}{ Treino A - RESISTÊNCIA } \\
\hline $\begin{array}{l}\mathrm{N}^{0} \text { de sessões } \\
\text { (semana) }\end{array}$ & Exercícios & № de séries & $\begin{array}{l}N^{0} \text { de } \\
\text { repetiçōes }\end{array}$ & $\begin{array}{c}\text { Variação de } \\
\text { carga }\end{array}$ \\
\hline \multirow[t]{2}{*}{1} & $\begin{array}{l}\text { Supino reto barra } \\
\text { guiada, crucifixo, } \\
\text { remada unilateral, } \\
\text { voador dorsal, } \\
\text { adutor. }\end{array}$ & \multirow[t]{2}{*}{3} & 15 & 6 a $50 \mathrm{Kg}^{*}$ \\
\hline & Panturilha & & 25 & Livre \\
\hline \multicolumn{5}{|c|}{ Treino B - RESISTÊNCIA } \\
\hline \multirow[t]{2}{*}{1} & $\begin{array}{l}\text { Elevação lateral, } \\
\text { elevação frontal, } \\
\text { tríceps corda, } \\
\text { rosca direta, rosca } \\
\text { concentrada, } \\
\text { tríceps pulley. }\end{array}$ & 3 & \multirow[t]{2}{*}{15} & 4a $15 \mathrm{Kg}^{*}$ \\
\hline & Abdominal infra & 4 & & Livre \\
\hline \multicolumn{5}{|c|}{ Treino C - RESISTÊNCIA } \\
\hline \multirow[t]{2}{*}{1} & $\begin{array}{c}\text { Avanço, cadeira } \\
\text { extensora, cadeira } \\
\text { flexora, legpress, } \\
\text { abdutor no } \\
\text { aparelho, glúteo } 4 \\
\text { apoios. }\end{array}$ & 3 & 15 & $10 \mathrm{a} 65 \mathrm{Kg}^{*}$ \\
\hline & Abdominal crunch & 4 & 20 & Livre \\
\hline \multicolumn{5}{|c|}{ Treino A - FORÇA } \\
\hline \multirow[t]{2}{*}{1} & $\begin{array}{l}\text { Agachamento, } \\
\text { cadeira extensora, } \\
\text { cadeira flexora, } \\
\text { avanço, abdutor } \\
\text { no aparelho. }\end{array}$ & \multirow[t]{2}{*}{4} & 6 & \multirow[t]{2}{*}{5 a $75 \mathrm{~kg}^{*}$} \\
\hline & Abdominal crunch & & 20 & \\
\hline \multicolumn{5}{|c|}{ Treino B - FORÇA } \\
\hline \multirow[t]{2}{*}{$\begin{array}{l}1 \\
1\end{array}$} & $\begin{array}{l}\text { Flexão de braço, } \\
\text { Pull over, Pulley } \\
\text { costas, Pulley } \\
\text { frente fechado, } \\
\text { Adutor no } \\
\text { aparelho. }\end{array}$ & \multirow[t]{2}{*}{$\begin{array}{l}4 \\
4\end{array}$} & 6 & \multirow[t]{2}{*}{$8 \mathrm{a} 60 \mathrm{Kg}^{*}$} \\
\hline & $\begin{array}{c}\text { Panturrilha no } \\
\text { aparelho }\end{array}$ & & 15 & \\
\hline
\end{tabular}

\begin{tabular}{|c|c|c|c|c|}
\hline \multicolumn{5}{|c|}{ Treino C - FORÇA } \\
\hline 1 & $\begin{array}{c}\text { Desenvolvimento, } \\
\text { Remada pegada } \\
\text { neutra, Rosca com } \\
\text { corda na polia, } \\
\text { Tríceps testa, } \\
\text { Remada alta, } \\
\text { Abdominal infra. }\end{array}$ & 4 & 6 & 6 a $15 \mathrm{Kg}^{*}$ \\
\hline & Tríceps repulsão & & & Livre \\
\hline
\end{tabular}

Legenda: ${ }^{\star K g}$ : kilograma. Fonte: dos autores

\section{Resultados}

Conforme o Gráfico 1, o grupo aula mix passou de um percentual de gordura geral de $28,91 \%$ para $25,50 \%$, com uma variação de $3,41 \%$ da avaliação pré-treino para pós-treino. Em relação ao resultado do grupo treinamento resistido, este passou de um percentual de gordura geral de $26,48 \%$ para $25,50 \%$, com uma variação de $0,98 \%$ da avaliação pré-treino para pós-treino.

Gráfico 1 - Percentual de gordura pré e pós-treino

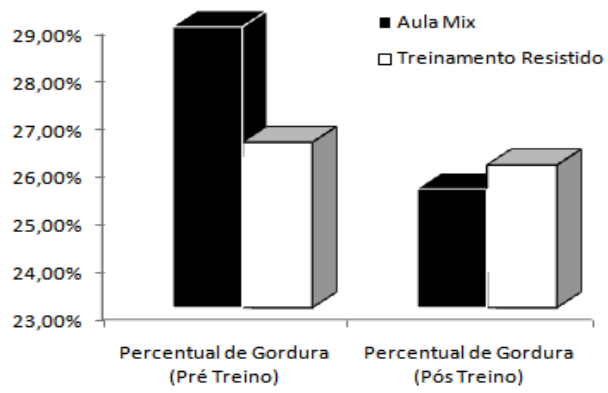

O Gráfico 2 revela o resultado da variável flexibilidade adquirida após a execução do programa de treinamento para os grupos, em que, com a modalidade aula mix obteve-se resultado pós-treino de $70 \%$ das participantes no nível de flexibilidade classificado como "excelente" (Tabela 2). Já no grupo treinamento resistido, obteve-se resultado de $60 \%$ pós-treino no nível de flexibilidade, este percentual também classificado como "excelente" (Tabela 2).

Gráfico 2 - Índice de flexibilidade pré e pós-treino

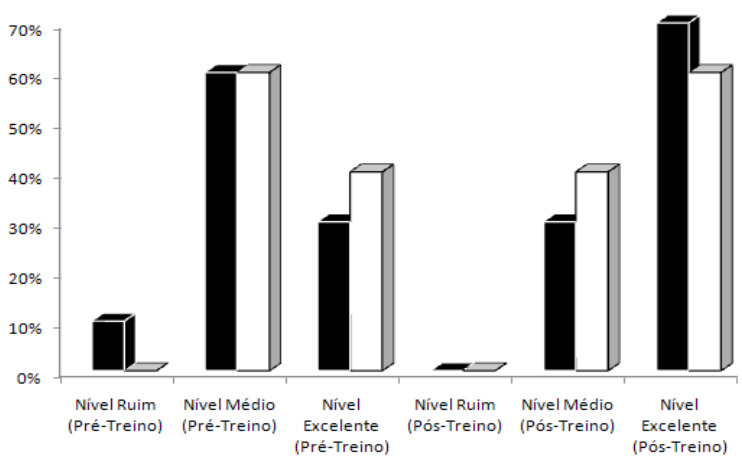


No Gráfico 3 é apresentada a análise do alcance do arremesso de peso de membro superior (Medicine Ball) entre os grupos. Percebe-se que o grupo aula mix (pré-treino) no nível "iniciante" contabilizou-se um resultado de $40 \%$ das participantes e no (pós-treino) deste mesmo nível, o percentual de participantes passou para 10\%. Já no grupo treinamento resistido no nível "iniciante" (pré-treino) contabilizou-se o resultado de $20 \%$ das participantes e no (pós-treino), nesse mesmo nível, nenhuma participante atingiu porcentagem significativa. Em relação ao nível "iniciante avançado” na aula mix (pré-treino), contabilizou-se um resultado de $40 \%$ das participantes, já (pós-treino) neste mesmo nível o percentual subiu para $70 \%$. No grupo treinamento resistido do nível "iniciante avançado", de $80 \%$ das participantes no (pré-treino) baixou para $60 \%$ no (pós-treino) neste mesmo nível. Contudo, no nível "intermediário" da aula mix (pré-treino) contabilizou $20 \%$ do grupo e no (pós-treino) manteve o mesmo resultado. Já no grupo treinamento resistido não contabilizou nenhuma participante no nível "intermediário" no (pré-treino), porém no (pós-treino) contabilizou $40 \%$ das participantes (Tabela 3 ).

Gráfico 3 - Alcance no teste do arremesso de peso

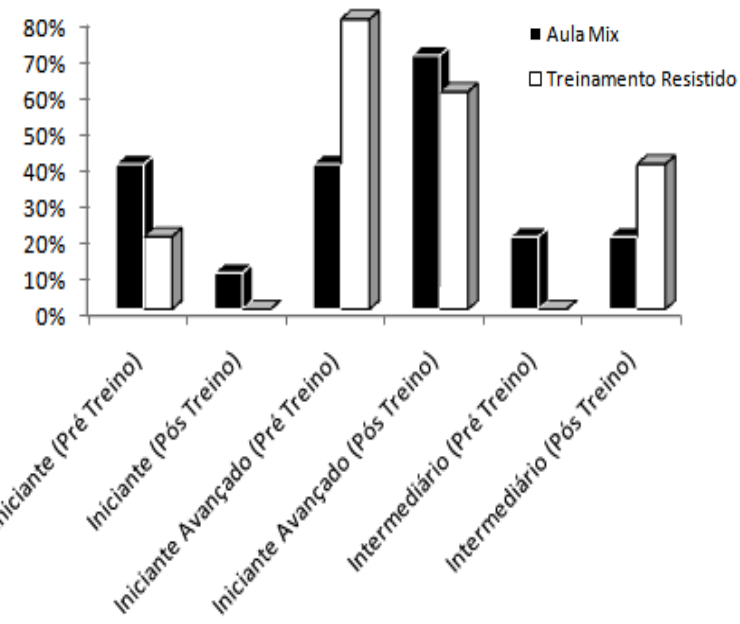

O Gráfico 4 traz a porcentagem das participantes em relação ao teste de salto vertical (força de membro inferior), em que o grupo aula mix obteve $10 \%$ de suas participantes no nível "abaixo da média” no (pré-treino) e no (pós-treino) nenhuma participante, em relação a este mesmo nível. O grupo treinamento resistido não obteve nenhuma participante no nível "abaixo da média”, tanto em relação ao pré-treino como no pós-treino. Em relação ao nível "médio" segundo a escala, o grupo aula mix (pré-treino) obteve 60\% das participantes e (pós-treino)
50\%. No grupo treinamento resistido, em relação ao nível "médio", no (pré-treino) contabilizou um resultado de $80 \%$ das participantes, no (pós-treino) 40\%. No nível "acima da média", aula mix (pré-treino) obteve 30\% de suas participantes neste nível, no (pós-treino) manteve o mesmo resultado. $\mathrm{O}$ grupo treinamento resistido, no nível “acima da média”, obteve $20 \%$ de suas participantes no (pré-treino), e no (pós-treino) subiu para 30\%. Contudo, no nível "excelente", ambos os grupos não obtiveram nenhuma participante neste nível no (pré-treino), porém no pós-treino obtiveram resultados iguais de $20 \%$ das participantes.

Gráfico 4 - Impulsão vertical

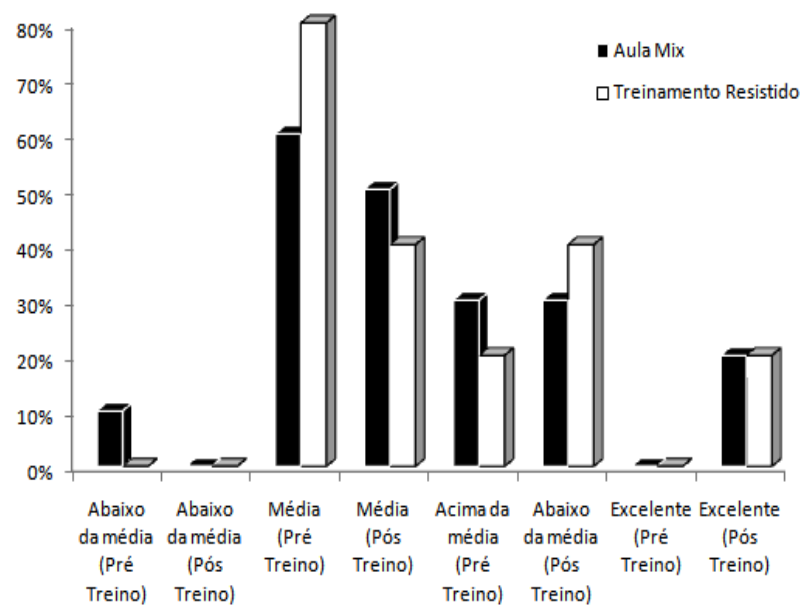

\section{Discussão}

De acordo com os resultados apresentados, é possível observar que ambas as modalidades, aula mix e treinamento resistido, apresentaram alterações positivas das variáveis de aptidão física que foram avaliadas: percentual de gordura, flexibilidade e força.

Em relação ao percentual de gordura percebemos que a modalidade aula mix obteve resultado melhor para perda de gordura geral em relação ao treinamento resistido, passando de um percentual de $28,91 \%$ para $25,50 \%$, queda de $3,41 \%$, $(\mathrm{p}=0,032)$, enquanto no treinamento resistido passou de um percentual de $26,48 \%$ para $25,50 \%$, em que a perda foi de $0,98 \%(\mathrm{p}=0,047)$, demonstrando nesse estudo, uma melhor resposta para a diminuição de gordura localizada na Aula Mix, o que corrobora os achados de Broeder et al. (1992), em que realizaram um estudo de três meses, utilizando treinamento aeróbio de baixa intensidade em comparação com o treinamento resistido. $\mathrm{O}$ grupo que se exercitou por meio de atividade 
aeróbia obteve perda de gordura, sem alterações na massa muscular, já o grupo que praticou treinamento resistido induziu tanto um aumento na massa muscular quanto uma redução na gordura corporal.

Porém, Kraemer et. al (1999), investigaram, durante três meses, três grupos de praticantes de exercício aeróbios e treinamento resistido, chegando à conclusão de que todos os participantes reduziram peso e que, dentre aqueles que optaram pelo treinamento resistido, $97 \%$ tiveram o peso perdido em gordura, já aqueles que optaram pelos exercícios aeróbios tiveram $78 \%$ do peso perdido em gordura, o que contradiz o atual estudo, em que a justificativa para tal resultado pode ser o maior tempo de treinamento, que foi de três meses, contra dois meses do presente estudo.

Outro estudo, como o de Gentil et al. (2007), mostra que os melhores resultados, em termos de modificação positiva na composição corporal (ganho de massa muscular e perda de gordura) são obtidos por meio do treinamento resistido, uma vez que, nessa modalidade, há o aumento da taxa metabólica em repouso (TMR), ou seja, quanto mais calorias são queimadas em repouso, mais fácil fica emagrecer.

Em relação à flexibilidade, também houve uma melhora acentuada do grupo aula mix em relação ao grupo treinamento resistido, em que o grupo aula mix melhorou seu nível denominado "excelente" de 30\% para $70 \%$, melhora de $40 \%$, $(p=0,029)$, enquanto o grupo treinamento resistido passou de $40 \%$ para $60 \%$ no nível "excelente", melhora de 20\%, ( $\mathrm{p}=0,037)$. Almeida et al. (2010), em um estudo em que foi avaliado a flexibilidade de praticantes de ginástica aeróbica, observaram maiores índices de flexibilidade, fato que foi explicado pela maior frequência de volume e exercícios de alongamento e flexibilidade no final das aulas, porém este estudo se difere do atual, pois, em ambas as modalidades (aula mix e treinamento resistido), houve sessões de alongamento antes e após as práticas.

$\mathrm{Na}$ valência de força, a modalidade treinamento resistido obteve melhores resultados do que a aula mix de uma forma geral, conseguindo concentrar maior número de participantes do estudo no nível "intermediário", cerca de $40 \%$ no pós-treino, $(\mathrm{p}=0,02)$, enquanto na aula mix manteve um número de apenas $20 \%$ neste nível $(\mathrm{p}=0,03)$. Isso corrobora os estudos de Conceição et al. (2003), ao apresentar o perfil dos níveis de força de membros superiores e inferiores e resistência muscular localizada (RML), de mulheres praticantes de ginástica localizada, que foram menores ao compararem os níveis de força dos membros inferiores e superiores de praticantes de treinamento resistido.

Já Nogueira et al. (2003) desenvolveram um estudo de comparação do nível de força de membros superiores e inferiores, grupo $1 \mathrm{com}(\mathrm{n}=16)$ idade média de 22,4 e o grupo 2 com ( $n=11$ ) idade média de 45,5, com mínimo de 6 meses de prática de ginástica localizada. O resultado não apresentou diferenças significativas nos níveis de força de membros superiores e inferiores entre os grupos, o que se assemelha aos resultados deste estudo, em que houve melhoras pouco significativas na modalidade aula mix comparadas aos resultados do treinamento resistido. Contudo no estudo de Nogueira et al. (2003), não houve comparações com a modalidade aula mix (exercícios aeróbicos).

Portanto, o treinamento resistido traz ganhos importantes, mas a corrida, ciclismo e outras atividades aeróbias, como as propostas pela Aula Mix, melhoram bastante o funcionamento do sistema cardiovascular e oferecem maior proteção contra os fatores de risco. Além disso, o exercício aeróbio também ajuda a emagrecer e a manter acelerado o metabolismo (HORTA; BARATA, 2006).

\section{Conclusão}

Conclui-se, por meio da reflexão proposta neste artigo, ao considerar a importância da atividade física para a saúde e, consequentemente, da prática de exercícios físicos aeróbios (aula mix) e anaeróbios (treinamento resistido), que, ao comparar as varáveis de aptidão física, percentual de gordura, flexibilidade e força, exercícios físicos do tipo aeróbio, foram mais eficientes quando o resultado esperado consistiu no aumento da flexibilidade ou na diminuição do percentual de gordura em comparação aos exercícios físicos do tipo anaeróbio, que, por sua vez, foram melhores em relação ao ganho de força.

Conclui-se que, independente da modalidade escolhida neste estudo (aula mix e treinamento resistido), e de acordo com os protocolos de treinamento propostos, as variáveis físicas percentual de gordura, flexibilidade e força apresentaram mudanças positivas em ambas as modalidades, o que corrobora o fato de que praticar exercícios físicos é a melhor garantia no que se refere à promoção de saúde e qualidade de vida. 


\section{Referências}

ALMEIDA, A. P. P. V.; VEVAS, R. P.; DOIMO, L. P. Avaliação do equilíbrio estático e dinâmico de idosas praticantes de hidroginástica e ginástica. Revista Brasileira de Cineantropometria e Desempenho Humano, Florianópolis, v. 12, n. 1, p. 55-61, jan./fev. 2010. doi: 10.5007/1980-0037.2010v12n1p55.

ANJOS, T. C. et al. Variáveis de condicionamento físico relacionado à saúde em adultas jovens submetidas a dois programas de atividade física: rebound exercise em solo. Fitness e Performance Journal, Rio de Janeiro, v. 1, p. 18-23, jan./fev. 2006.

BARATA, T. et al. Actividade física e medicina moderna. Odivelas: Europress, 1997.

BITTENCOURT, N. Treinamento resistido: uma abordagem tecnológica. Rio de Janeiro: Sprint, 1986.

BROEDER, C. E, et. al. The effects of either high-intensity resistance or endurance training on resting metabolic rate. The American Journal of Clinical Nutrition, Bethesda, v. 55, n. 4, p. 802-810, Apr. 1992.

CANADIAN MINISTRY OF STATE, FITNESS AND AMATEUR SPORT. Canadian Standardized Test of Fitness. Ottawa: Government of Canada, Fitness and Amateur Sport, 1987.

CARVALHO, T. C. et al. Normatização dos equipamentos e técnicas de reabilitação cardiovascular supervisionada. Arquivo Brasileiro de Cardiologia, São Paulo, v. 83 , n. 5, p. 448-452, nov. 2004. doi: 10.1590/S0066$782 \mathrm{X} 2004001700012$.

CONCEIÇÃO, M. C. S. et al. Perfil do nível de força e RML em mulheres praticantes de ginástica localizada. In: SIMPÓSIO INTERNACIONAL DE CIÊNCIA DO ESPORTE, 26., 2003, São Paulo. Anais... São Paulo: CELAFISC, 2003. p. 196.

FLECK, S.; KRAEMER, W. Fundamentos do treinamento de força muscular. São Paulo: Artmed, 2006.

GENTIL, P.; OLIVEIRA, E.; ROCHA JUNIOR, V. A.; CARMO, J. Effects of exercise order on upper-body muscle activation and exercise performance. Journal of Strength and Conditioning Research, Champaign, v. 21, n. 4, p. 1082-1086, nov. 2007.

GONÇALVES, A.; VILARTA, R. Qualidade de vida e atividade física: explorando teorias e práticas. Barueri: Manole, 2004.
HOLLMANN, W.; HETTINGER, T. H. Medicina do esporte. São Paulo: Manole, 1983.

HORTA, L.; BARATA, T. Actividade física e prevenção primária das doenças cardiovasculares. Ludens, Lisboa, v. 15, n. 3, p. 24-28, jul./set. 1995.

KRAEMER, W. J. et al. Effects of heavy-resistance training on hormonal response patterns in younger vs. older men. Journal of Applied Physiology, Bethesda, v. 87, n. 3, p. 982-992, set. 1999.

MARINS, J. C. B.; GIANNICHI, R. S. Avaliação e prescrição de atividade física: guia prático. Rio de Janeiro: Shape, 1998.

MATSUDO, V. Exercícios resistidos. Revista Âmbito Medicina Desportiva, São Paulo, v. 2, n. 27, p. 24-26, jan. 1997.

NOGUEIRA, D. T. et al. Estudo comparativo do nível de força e de RML em mulheres praticantes de ginástica localizada. In: SIMPÓSIO INTERNACIONAL DE CIÊNCIA DO ESPORTE, 26., 2003, São Paulo. Anais... São Paulo: CELAFISC, 2003. p. 153.

POLLOCK, M. L.; ROCHA, M. L.; WILMORE, J. H. Exercícios na saúde e na doença: avaliação e prescrição para prevenção e reabilitação. Rio de Janeiro: Medsi, 1993.

SANTARÉM, J. M. O exercício. São Paulo: Atheneu, 1999.

SANTOS, L. B.; FISCHER, L. M. Efeitos do treinamento Resistido e o intervalo ativo na composição corporal em mulheres de 18 a 35 anos. 2007. s.f. Trabalho de Conclusão de Curso (Especialização) - Universidade Gama Filho, Florianópolis, 2007.

UCHIDA, M. C. et al. Efeito de diferentes protocolos de treinamento de força sobre parâmetros morfofuncionais, hormonais e imunológicos. Revista Brasileira de Medicina do Esporte, São Paulo, v. 12, n. 1, p. 65-73, jan./fev. 2006. doi: 10.1590/S1517-86922006000100005.

VILLALBA, C. B. Manual de cycle indoor. Barcelona: Pai do Tribo, 2005.

WEINECK, J. Biologia do esporte. Barueri: Manole, 2005.

WELLS, K. F; DILLON, E. K. The sit and reach: a test of back and leg flexibility. Research Quarterly for Exercise and Sport, Washington, v. 23, n. 1, p. 115-118, mar. 1952. doi: 10.1080/10671188.1952.10761965. 